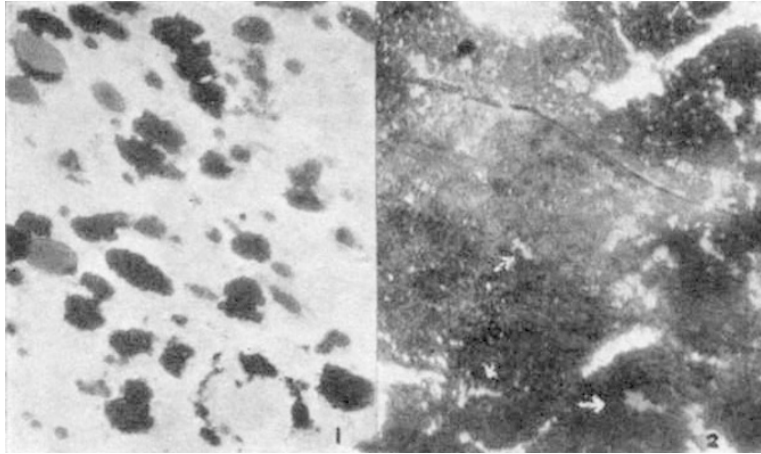

Because of the persistence of different colours of the coal ingredients in the anthracites when treated this way, a quantitative estimation of the vitrinite, fusinite, exinite fraction of the coal of all colliery dusts should now be possible. The mineral fraction is readily estimated by the areas of translucency, and it is hoped, at least, to separate the minerals into several groups by a further development of the method based on interference phenomena.

Medical Research Council

R. G. H. B. BODDY

Pneumoconiosis Research Unit (S. Wales),

Llandough Hospital,

Penarth, Glam.

Nov. 3.

I Boddy, R. G. H. B., Nature, 151, 54 (1943); Fuel, 22, 56 (1943); "Ultra-fine Structure of Coals and Coke"' (Brit. Coal Util. Res. Assoc., 1943).

2 Bangham and Berkowitz, Research, 86 (1947).

\section{Alleged Optical Activity in Crystal Class $m$}

OptICAL activity is theoretically possible in crystals belonging to the non-enantiomorphous classes $m$, $m m 2, \overline{4}$ and $\overline{42} \mathrm{~m}$; but only one substance has ever been cited as an examplex. This is the dimer of the $\beta$-form of methyl mesityl oxide oxalate $\left(\mathrm{C}_{8} \mathrm{H}_{12} \mathrm{O}_{4}\right)_{2}$, which was assigned by Sommerfeldt ${ }^{2}$ to class $m$ on the basis of morphological measurements.

Crystals corresponding exactly with those described by Sommerfeldt have been examined with X-rays and found to have the uniquely identifiable space group $P 2, c$ with $a=9 \cdot 15+3 \mathrm{~A} ., b=11 \cdot 56+5 \mathrm{~A}$., $c=18.02 \pm 5$ A., $\beta=96^{\circ} 12^{\prime} \pm 20^{\prime} ; d_{\text {obs. }}=1 \cdot 290$ gm.cm. ${ }^{-3}, d_{\text {calc. }}=1 \cdot 289 \pm 10$ gm.cm..$^{-3}$ for four molecules per unit cell. Sommerfeldt's axes are related to these by the transformation $102 / 010 / \overline{1} 02$. Polysynthetic twinning is prevalent, though the polarizing microscope gives no hint of this: the twins have $(10 \overrightarrow{2})$ and $[010]$ in common and the twinned reciprocal lattice approximates closely to Laue symmetry $\mathrm{mmm}$. The habit of the single crystals resembles that of the crystals of the corresponding ethyl ester, which Sommerfeldt assigned to class $2 / \mathrm{m}$. (The lattice of the ethyl ester apparently does not so easily permit analogous polysynthetic twinning.) The assignment of the methyl ester to class $m$ is therefore erroneous though perhaps understandable.

sommerfeldt's evidence for optical activity, which was only qualitative, may now be rejected for two reasons : $(a)$ the centrosymmetry of the space group ; and $(b)$ his observations could, as Voigt ${ }^{3}$ pointed out, be the result of twinning, or merely a consequence of increasing the illumination and so forcing a perceptible amount of light through the crossed nicols. This example may therefore be rejected, as it neither exhibits optical activity nor is in class $m$.

The absence of any example of optical activity in classes $m, m m 2, \overline{4}$ and $\overline{4} 2 m$ may suggest some development of the existing theory or, more probably, reflects the considerable experimental difficulties of measuring optical activity in the presence of birefringence, and the decline of interest in this subject during recent years. A large number of substances have by now been reliably assigned to these classes, so that an intensive search among suitable representatives seems desirable.

A full account of the crystallography of the monomeric and dimeric forms, and a detailed discussion of Sommerfeldt's interpretation of the optical observations, will be published elsewhere.

Physics Department,

D. ROGERS

University College,

Cardiff. Oet. 31 .

' Wooster, W. A., “Crystal Physics”, 154 (Camb. Univ. Press, 1938) 2 Sommerfeldt, E., "Neues Jahrbuch für Mineralogie", 1, 58 (1908). ${ }^{3}$ Yoigt, W., Phys. Z., 7, 267 (1906).

\section{Langmuir Adsorption as a Determinant of Conductivity in a Low-frequency Electric Discharge}

The marked influence of sorption of electronegative gases on photo- and thermo-electric currents in a number of systems is well known'. We have now investigated the significance of Langmuir adsorption or chemi-sorption, chiefly occurring under discharge ${ }^{2}$, in determining the conductivity of a gas excited at reduced pressures by low-frequency potentials.

Siemens-type ozonizers made of soft soda glass and degassed for the removal of pre-adsorbed gases by heating for $15 \mathrm{hr}$. at $200^{\circ} \mathrm{C} .{ }^{3}$ were employed; they were filled with iodine or water vapour at a desired pressure and excited by a 50-cycle potential. The root-mean-square value of the current $i$ was measured by a microammeter or a galvanometer actuated by a crystal detector, vacuo-junction, etc.; and the nature of the current was studied by a cathoderay oscillograph. The accompanying graph gives a typical series of results.

With the time of exposure to discharge due to a fixed applied potential sufficient to cause a self-

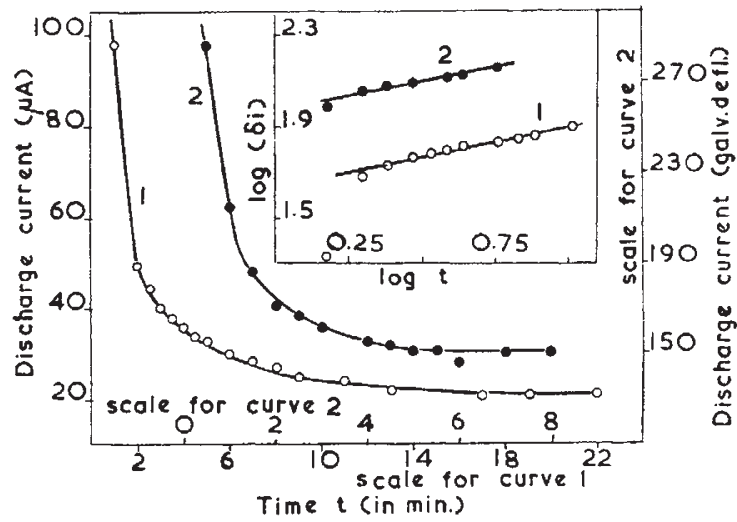

Variation of the discharge current with time of exposure to discharge. Curve (1), in water; curve (2), in iodine vapour 